\title{
Gene drives in malaria control: What we need to know
}

\author{
Reagan Mudziwapasi ${ }^{1}$, Munyaradzi Christopher Changara ${ }^{1}$, Abigarl Ndudzo ${ }^{1}$, Tinotenda \\ Kaseke $^{1}$, Farai Godobo ${ }^{1}$, Ryman Shoko ${ }^{2}$, Sakhile Ndlovu ${ }^{1}$, Fanuel Songwe ${ }^{1}$, and Sandra \\ Sibonani Mlambo ${ }^{1}$ \\ ${ }^{1}$ Affiliation not available \\ ${ }^{2}$ PREreview
}

May 6, 2020

\section{Gene drives in malaria control: What we need to know}

\author{
${ }^{a}$ Reagan Mudziwapasi*, ${ }^{\mathrm{b}}$ Munyaradzi Christopher Changara, ${ }^{\mathrm{a}}$ Abigarl Ndudzo, ${ }^{\mathrm{b}}$ Tinotenda Kaseke, Farai \\ Godobo, ${ }^{\mathrm{c}}$ Ryman Shoko, ${ }^{\mathrm{d}}$ Fanuel Songwe, ${ }^{\mathrm{a}}$ Sakhile Ndlovu and ${ }^{\mathrm{b}}$ Sandra Sibonani Mlambo \\ a Department of Crop and Soil Sciences (Applied Biotechnology Program), Lupane State University, P. O. \\ Box 170, Lupane, Zimbabwe \\ ${ }^{\mathrm{b}}$ Department of Biotechnology, Chinhoyi University of Technology, P. O. Box 7724, Chinhoyi, Zimbabwe \\ ${ }^{\mathrm{c}}$ Department of Biology, Chinhoyi University of Technology, P. O. Box 7724, Chinhoyi, Zimbabwe \\ ${ }^{\mathrm{d}}$ Department of Biosciences and Biotechnology, Midlands State University, P. Bag 9055 Senga Road, Gweru, \\ Zimbabwe
}

*Corresponding author: reaganmudziwapasi@yahoo.com

\begin{abstract}
Gene drives are being used to enhance a DNA sequence's likelihood of passing between generations via sexual reproduction. Gene drives can manipulate natural populations. They can be used to suppress populations by reducing the number of individuals in a population. Some of the more than 3000 mosquito species in the world are vectors of diseases. Malaria is a typical disease whose vectors are mosquitoes. It affects mostly tropical countries. It kills many people annually many of whom are children. Interventions currently in use such as indoor residual spraying and mosquito nets are insufficient. Gene drives can be used in different ways to control mosquito populations or to eliminate mosquito species thereby reducing malaria cases and deaths. This can be through population replacement or suppression. However, before the elimination of any mosquito species for malaria control it is necessary to consider the effects of such an action. Additionally, there is a need to review the options available for the control of mosquitoes and to create awareness on the benefits and risks of such an action. This paper, therefore, looks at the role of mosquitoes in the environment, methods of controlling mosquitoes and malaria and necessary considerations when using gene drives inter alia.
\end{abstract}

Keywords: gene-drive, malaria, mosquito control

\section{Introduction}


The current increase in genetic modification techniques and their applications require us to carefully advance science while navigating uncertainty and aligning our research with public values. There is a need to understand the science behind these new technologies to cover knowledge gaps, be ethical, and manage the risks pertaining to their applications. An example of such technologies is the 'gene drives. These are a genetic engineering technology that propagates a particular set of genes throughout a population. Gene drives ensure biased inheritance and enhance a DNA sequence's likelihood of passing between generations via sexual reproduction by more than the natural $50 \%$ (Noble et al., 2018). They bias the inheritance of desired traits by cutting a wild-type allele and copying the drive system in its place (Esvelt et al., 2014). Gene drives can be used to suppress populations by reducing numbers of individuals in a population thereby resulting in changes in gene frequencies within a population. Global gene drives will spread throughout all populations of a species connected by gene flow and persist (Beaghton et al., 2016).

The principle of gene drives is not a new phenomenon as there are cases of the mechanism occurring naturally. For example, a gene can produce multiple copies of itself in a genome, or a gene disabling other genes to increase inheritance odds. Gene drive-modified organisms hold the potential of either yielding great benefits or harmful ecological changes. For example, gene drives have the potential to offer a high-impact, costeffective, and durable method of controlling mosquito populations (James et al., 2018). The common gene drive systems include the maternal effect dominant embryonic arrest system, homing-based drives using homing endonuclease genes, under-dominance or heterozygote inferiority drives, sex-linked meiotic drives, and heritable microorganisms. Homing for gene knock-outs is a particularly simple mechanism of achieving drive based on the activity of endonucleases. A homing-based RNA-guided drive may be removed from a population by designing a reversal drive encoding a gRNA that targets the previous generation's drive. A homing drive may be utilized to suppress a population by homing into a critical gene, the disruption of which induces recessive sterility or lethality (Marshall et al., 2017).

The novelty of recent gene drives resides in the use of the CRISPR technique, which not only allows gene editing with precision, speed, and economy but also has the potential of ensuring that alterations made in wild organisms will persist in nature.CRISPR-Cas9 systems can be precisely used to alter DNA regions in order to yield a gene drive (Champer et al., 2016). CRISPR gene drives make use of CRISPR's DNA revision mechanism by copying engineered nucleotide sequences into homologous chromosomes, thereby guaranteeing the inheritance of edited genes in all offspring (Tuna et al., 2019). CRISPR based gene drives can spread genes particularly rapidly because their components can be tailored to replace alternative copies of a targeted gene. This will ensure that only desired versions of genes are passed on to offspring. Self-propagating gene drives are designed so that they can always spread as long as there are wild organisms around while selfexhausting gene drives lose their ability to spread over time. Self-propagating CRISPR based gene drives can spread if a few organisms with the gene drive elements are released into the wild (Noble et al., 2018).

In this paper, we discuss the methods that are used in mosquito control in malaria programs and how gene drives can be used to aid them in the control of mosquito populations. We also discuss the benefits of mosquitoes and their negative impacts on the environment.

\section{Role of mosquitoes in the ecosystem and to human health}

There are currently more than 3000 mosquito species in the world grouped into 39 genera and 135 subgenera (Crans, 2004). Mosquitoes exist at the bottom of the food chain and have a role in the aquatic food chain necessary in maintaining a natural balance. Mosquito larvae filter feeder which feeds on unicellular algae and other tiny organic particles thus clearing the water body of debris (Waldbauer, 1998). The larvae, in turn, serve as food for the aquatic community for fish, frogs, and tadpoles. Their role on the bottom of the food chain passes the larval stage. They serve as prey for birds, bats, and spiders, salamanders, 
spiders, lizards, turtles, dragonflies, swallows, and bats. The mosquito eggs are robust and survive the harsh weather, hatching when the snow melts thus providing food for migratory birds especially in Arctic tundra (Lundkvist et al., 2003). Mosquitoes depend on nectar for energy and thus act as pollinators thus ensuring, mainly aquatic plants to thrive. An example is the swamp orchids such as Habenaria obtusata to which genus Aedes have specialized as pollinators

Mosquitoes are known to be a vector for the transmission of several diseases known as mosquito-borne viruses (moboviruses). These moboviruses include yellow fever, West Nile virus (Hubálek and Halouzka, 1999), dengue fever (Rueda, 2004), filariasis, Zika flavivirus (Kindhauser et al., 2016), Chikungunya (Weaver and Lecuit, 2015) and other arboviruses (Gubler, 2001). They also carry malaria-causing parasites such as Plasmodium vivax and P. falciparum. Malaria has ravaged the Sub-Saharan region for over 300000 years causing about 438000 deaths each year (Cartolovni, 2017). Despite their well-documented role in disease transmission, mosquitoes have important direct roles to humans. Mosquito saliva has been explored for medicinal properties in cardiovascular disease and was found to produce anticoagulant factors (Stark and James, 1998). There is thus the potential for the development of anticlotting drugs, such as clotting inhibitors and capillary dilators from the molecules found in the insect's saliva. (Derbie, 2019). Also, mosquito bites have been associated with the modulation of host immune response(Schneider and Higgs, 2008). However, despite their potential usefulness to human health, there is still debate about their ultimate importance. Part of the scientific world suggests an eradication of certain species of mosquito responsible for the transmission of diseases with the hope that there will be no significant negative effect on the environment.

Mosquitoes that can carry plasmodium are of increasing interest in cancer treatment. This is because experimental results show that infection with some species of plasmodium can help stimulate the immune system to better fight cancers such as Hepatocellular carcinoma (HCC). HCC accounts for between 85 and $90 \%$ of primary liver cancers. It is the third most common causes of cancer mortality worldwide. Plasmodium yoelii 17XNL infection significantly suppresses Lewis lung cancer (LLC) cell growth. This occurs via induction of innate and adaptive antitumor responses. Additionally, plasmodium infection inhibits tumor angiogenesis (Yang et al., 2017; Chen et al., 2011). Thus, although seemingly not important, mosquitoes that carry plasmodium can be used to help find ways to treat cancer. Thus, it is risky to eliminate whole species especially globally. Understanding mosquitoes is therefore a potential source of treatment methods and drugs which can turn out into billion-dollar industries and save millions of lives.

\section{Methods of controlling or eradicating mosquitoes and malaria}

Out of 460 different Anopheles species, 30 to 40 are vectors for the Plasmodium parasite. Methods for the control and treatment of malaria have been relatively successful, as evidenced by the decreasing malarial deaths (Cartolovni, 2017). The methods include vector control through insecticide-treated mosquito bed nets and indoor residual spraying. These have helped decrease the presence of Anopheles mosquitoes (Eckhoff et al., 2016; Bhatt et al., 2015). However, there is still residual transmission from mosquito vectors that feed outdoors or early in the evening. Malaria is treatable with anti-malarial drugs, which helps prevent the spread of the parasite by decreasing the number of parasites in the blood. The problem with vector control and treatment of malaria is the emergence of insecticide and drug resistance. Both the Anopheles mosquitoes and the Plasmodium parasite are showing resistance to the most commonly used chemical and pharmaceutical options to fight malaria such as pyrethroid. Artemisinin combination therapies (ACTs) are key to the treatment of $P$. falciparum malaria throughout the malaria endemic world. The emergence and geographic spread of artemisinin resistant $P$. falciparum represent a serious threat to global malaria control and to aspirations to eliminate malaria. Resistance is attributed to mutations of the PfKelch13 gene. The gene has multiple independent origins across the Greater Mekong sub-region, which has motivated a regional malaria elimination agenda (Mernad et al., 2016). There are multiple mechanisms of insecticide resistance including changes to insecticide target molecules that render the insecticide unable to bind, behavioral changes leading 
to the avoidance of insecticide contact, thickening of the insect's cuticle to prevent the insecticide reaching its target and detoxification of the insecticide before it reaches its target (metabolic resistance) (Barnes et al., 2017) ). In the malaria vector An. funestus, pyrethroid resistance is mainly conferred by metabolic resistance associated with a major quantitative trait locus (QTL) at which two duplicated cytochrome P450 monooxygenases (CYP6P9a and CYP6P9b) are the main resistance genes (Wang and Jacobs-Lorena, 2013). The resistance to insecticides means that Malariaeradication requires new tools in addition to those currently deployed.

When mosquitoes take in a blood meal, the blood contains excess salts, such as potassium chloride that needs to be excreted via the kidney (Mernad et al., 2016). A team of researchers from Vanderbilt University Medical Center and Ohio State University developed a new class of insecticides that target the mosquito kidney. About 26000 compounds were screened for their ability to inhibit a potassium channel, Kir1, involved in urine production. It was noted that a compound called VU041 rapidly blocked the Kir1 channel activity (Pike et al., 2017). It is specific to mosquitoes and does not affect any mammalian potassium channels tested. The team monitored mosquitoes to assess kidney function and observed that when untreated mosquitoes consumed a blood meal, their abdominal diameter immediately doubled, and then decreased over the next 24 hours. In contrast, the abdominal diameters of mosquitoes treated with VU041 increased but did not decrease, suggesting that the impairment of kidney function. The mosquitoes kept on increasing in weight until they burst. VU041 was found to reduce egg laying after blood feeding suggesting that VU041 can be used to control mosquito populations.

A recently developed strategy is to use Metarhizium anisopliae, a fungus that naturally attacks mosquitoes, as mosquito-specific biopesticides (Lovetteet al., 2019). The mosquitoes must acquire the fungus soon after becoming infected with the malaria parasite. Rather than developing fungi that rapidly kill the mosquito, the fungus is genetically modified to block Plasmodium development inside the mosquito. Metarhizium pingshaense provides an effective, mosquito-specific delivery system for potent insect-selective toxins. After invading a mosquito, the transgenic fungi produce small molecules such as the human anti-malarial antibody and a scorpion antimicrobial toxin (Pike et al., 2017). When mosquitoes that are heavily infected with $P$. falciparum are sprayed with transgenic fungi, they have a significantly reduced parasite development. The transgenic fungus does not significantly affect mosquito survival when compared to the wild-type fungus. Hence the transgenic fungi do not lead to rapid mosquito resistance when used in the field.

Changing the mosquito's ability to support the life cycle of Plasmodium parasites can be achieved through genetic engineering of the mosquito gut, making it too hard for Plasmodium to survive (Wang et al., 2013). One way of achieving that is is to genetically modify the Anopheles mosquito to make part of the mosquito's gut, where the Plasmodium parasite normally grows, into an inhospitable habitat where Plasmodium cannot survive. This means that the mosquitoes will need to have new effector genes introduced so they can be expressed as anti-Plasmodium proteins within the mosquito's gut, making the environment uninhabitable for the Plasmodium to survive. Introduction of the effector genes into the mosquito includes using fungi or viruses that already infect mosquitoes or using bacterial symbionts that already inhabit the mosquito gut (Carvalho et al., 2015). Another way is to modify the symbiotic gut bacteria. When the modified bacteria enter the mosquito gut, the mosquito begins to express the anti-Plasmodium proteins, making the mosquito gut inhospitable to Plasmodium parasites (Pike et al., 2017).

One of the recent technologies of genetic engineering to control malaria involves decreasing the mosquito population so there are fewer mosquitoes to transmit malaria. The genetic modification of the mosquito vector employs CRISPR technologies while the dissemination of the sterility gene to run in the populations 
uses the gene drive technology (Tuna et al., 2019). An example of a potential method has been successfully tested in Aedes mosquitoes to combat dengue. The approach involves genetically modifying male mosquitoes so their offspring never matured, dying before they were able to transmit dengue (Carvalho et al., 2015). The use of these transgenic sterile males resulted in a wild mosquito population decrease of $80-95 \%$. The study showed that genetically engineering sterile mosquitoes can drastically decrease the mosquito population in an area.

Mosquitoes can be genetically modified to alter the expression of their anti-Plasmodium immune genes in a population with wild-type mosquitoes. Multiple GM Anopheles stephensi lines can be created that are resistant to Plasmodium falciparum due to the up-regulation of mosquito immune genes in the midgut or fat body after a blood meal, using the carboxypeptidase (Cp) or vitellogenin (Vg) promoter, respectively (Mernad et al., 2016). These strains will possess elevated anti-Plasmodium and antibacterial activities through either the immune-deficiency pathway-associated NF- $\varkappa \mathrm{B}$ transcription factor Rel2 or the Down syndrome celladhesion molecule (AgDscam) splice form AgDsPf (Swale et al., 2016). The GM lines can be backcrossed with the original wild-type stock for five generations and be continually reared under the same conditions to ensure the same genetic and environmental background. Genetically modified mosquitoes with increased immune activity in the midgut tissue will not have an observed fitness disadvantage and will show reduced microbial loads in both the midgut and reproductive organs. These changes result in a mating preference of genetically modified males for wild-type females, whereas wild-type males will prefer genetically modified females. These changes will foster the spread of genetic modification in a mosquito population and help control mosquito populations (Pike et al., 2017).

\section{Potential of Gene drives in eradicating mosquitoes and malaria}

Gene drives can be characterized by the rate of spread, species specificity, fitness cost, susceptibility to resistance, removability, and reversibility. Engineered gene drives can be divided into the modification drive types designed to spread genomic changes and or genetic payloads throughout a population, suppression drive types designed to reduce or eliminate the population of its target organism and reversal gene drive types which induce further changes that may undo a phenotypic alteration caused by the initial gene drive. The envisioned goal for applying gene drives is to reduce or eliminate vector mosquito populations or to render them less competent to transmit pathogens. With a gene drive, not only is it possible to alter an organism's gene, but it is also possible to insert in the genome the Clustered Regularly Interspaced Short Palindromic Repeats (CRISPR) copy-paste system which includes the gRNA and Cas protein. This allows the gene alteration to self-replicate in subsequent generations. As an example, when an altered mosquito mates with a wild mosquito, the offspring receives an altered chromosome and a wild chromosome from each parent. The CRISPR system inherited from the altered parent will cut the wild gene inherited from the wild parent and copy the altered gene into the offspring's genome along with the gene drive. The offspring then carries two copies of the altered gene, ensuring its transmission to the next generation. When a new generation of altered mosquito mate with the wild types, the process will repeat itself allowing the alteration and the gene drive to spread in the whole population. The gene drive, therefore, appears to be a reliable mechanism for propagating altered genes, which in theory would allow gene alterations to persist in nature and permanently change the target population and possibly an entire species. Computational modeling based on other gene drive systems suggests that the type of drive that can be achieved with the CRISPR/Cas9 system can be so effective that release of low numbers of modified mosquitoes into the environment could result in establishment of the genetic modification in the natural interbreeding population (Tuna et al., 2019; Eckoff et al., 2016).

One way of applying gene drives in mosquito control is via the use of the Wolbachia gene drive. Wolbachia is a naturally occurring bacterium that was previously found to block the development of Plasmodium parasites 
in mosquitoes. Wolbachia can be transmitted by an infected female insect to the offspring. Uninfected females that mate with infected males rarely produce viable eggs, a reproductive dead end that gives infected females a reproductive advantage and helps the bacteria spread quickly. Wolbachia were successfully used in a field trial to control dengue, another mosquito-borne disease (Swale et al., 2016). However, the bacteria do not pass consistently from mother to offspring in Anopheles mosquitoes, which spread malaria. The researchers injected a strain of Wolbachia derived from another type of mosquito into A. stephensi embryos. Once matured, the adult females mated with uninfected male mosquitoes to create a stable Wolbachia infection that persisted for 34 generations (the end of the study period). Uninfected females rarely produced viable eggs with infected males. The researchers found that Wolbachia infection reduced the number of malaria parasites in both the mosquito midgut and salivary glands. Wolbachia infection causes the formation of reactive oxygen species, which inhibit parasite development.

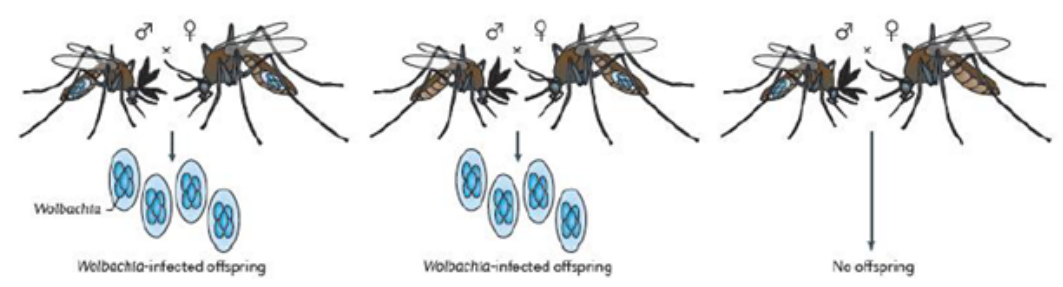

(Champer, 2016)

Figure 1: Shows how Wolbachia gene drives can eliminate mosquito species.

Male killing strains of Wolbachia can be used for population suppression. However, they have a moderate rate of spread, the resistance allele generation rate is unknown and they cannot be reversed.

Standard drives are designed to genetically alter an entire species without setting any temporal or geophysical limitations to their spread (Figure 2). For instance, Kyrou et al. (2018) have already demonstrated a method of engineering a construct targeting the Agdsx gene which led to "total population collapse" in caged mosquitoes. Daisy drive systems, also known as split drives, instead contain split up portions of CRISPR that require sequential action for the drive to operate: element A drives element B, which drives element C, and so on until the final element, which does not drive any others (Tuna et al., 2019). 

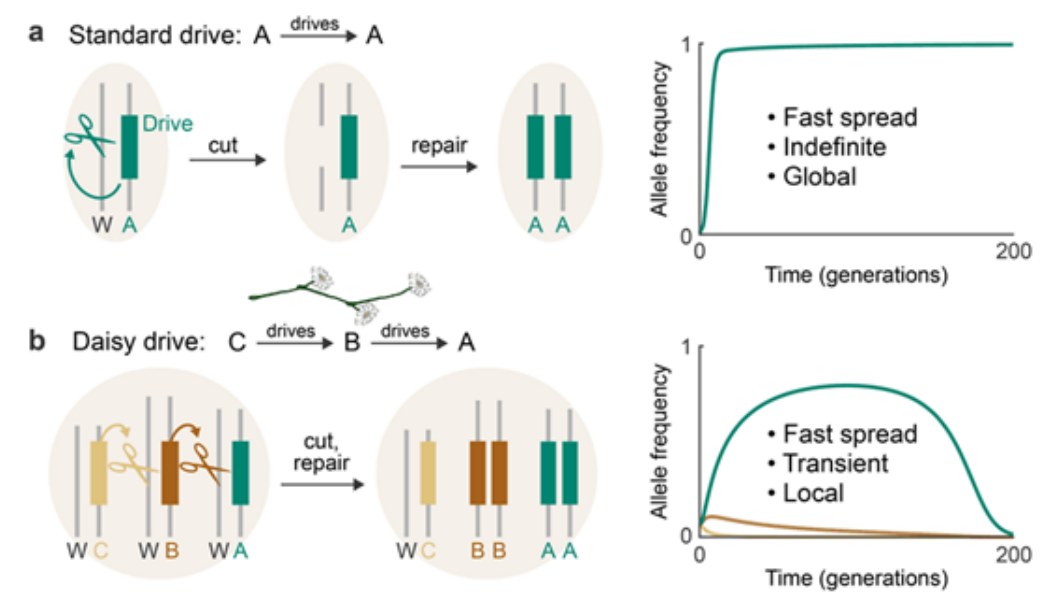

(Noble et al, 2019)

Figure 2: Standard and Daisy gene drives

Despite these promising theoretical results, current technological limitations preclude the safe use of daisy drive elements. Specifically, any recombination event that moves one or more guide RNAs within an upstream element of the chain into any downstream element will convert a linear daisy drive chain into a self-sustaining gene drive 'necklace' anticipated to spread globally (Noble et al., 2019; Scudellari, 2019).

Other types of drives include;

i) Precision drives which directly alter genomes so that the effects of the drive are only realized in targeted organisms,

ii) Alteration drives which make specific changes, either by adding or by making edits to genes,

iii) Suppression drives that reduce the number of organisms in a population, often using methods such as reduction of vectorial capacities,

iv) Immunising drives which prevent the spread of unwanted genes by pre-emptively altering genetic sequences to block the effects of precision drives (Tuna et al.,2019).

These drives can be used to add genes that cause sterility in mosquitoes and to reduce the number of mosquitoes with the capacity to transmit plasmodium. This will consequently reduce the population of vectors of the malaria parasite and hence malaria cases.

Homing endonuclease genes (HEGs) may be designed to manipulate populations by targeting other suitable genes, such as genes to reduce lifespan, to bias sex ratios, to impede host-seeking, to block pathogen development, or to block the ability of the modified organism to act as a vector for pathogens. In mosquitoes, a synthetic version of the homing reaction was first demonstrated in A. gambiae (Windbichler et al. 2011) using a homing endonuclease from yeast. Conceptually, the simplest use for homing is to produce a populationwide gene knock-out. Modeling shows that if the knock-out phenotype is recessive and if the homing reaction is confined to the germline, then it is even possible for a homing endonuclease that causes lethality or sterility to increase in frequency in a population, potentially suppressing the population as it does so (North, and Burt 2017). Another possibility is to disrupt malaria transmission by targeting genes needed for the Plasmodium parasite to invade into, develop within, or exit out of the mosquito vector. One review lists 38 genes that when knocked-down show some reduction in oocyst number or sporozoite count (Sreenivasamurthy et 
al. 2013), and some of these genes may be suitable for this approach. For the homing reaction to lead to preferential inheritance of the enzyme construct, the enzyme must be expressed in the germline. Thus far, promoters used for this in Anopheles have been from the B2- tubulin gene (Catteruccia, Benton, and Crisanti 2005) and from the vasa gene (Papathanos et al., 2009). Mosquito population control using homing into female infertility genes is illustrated in Figure 3.
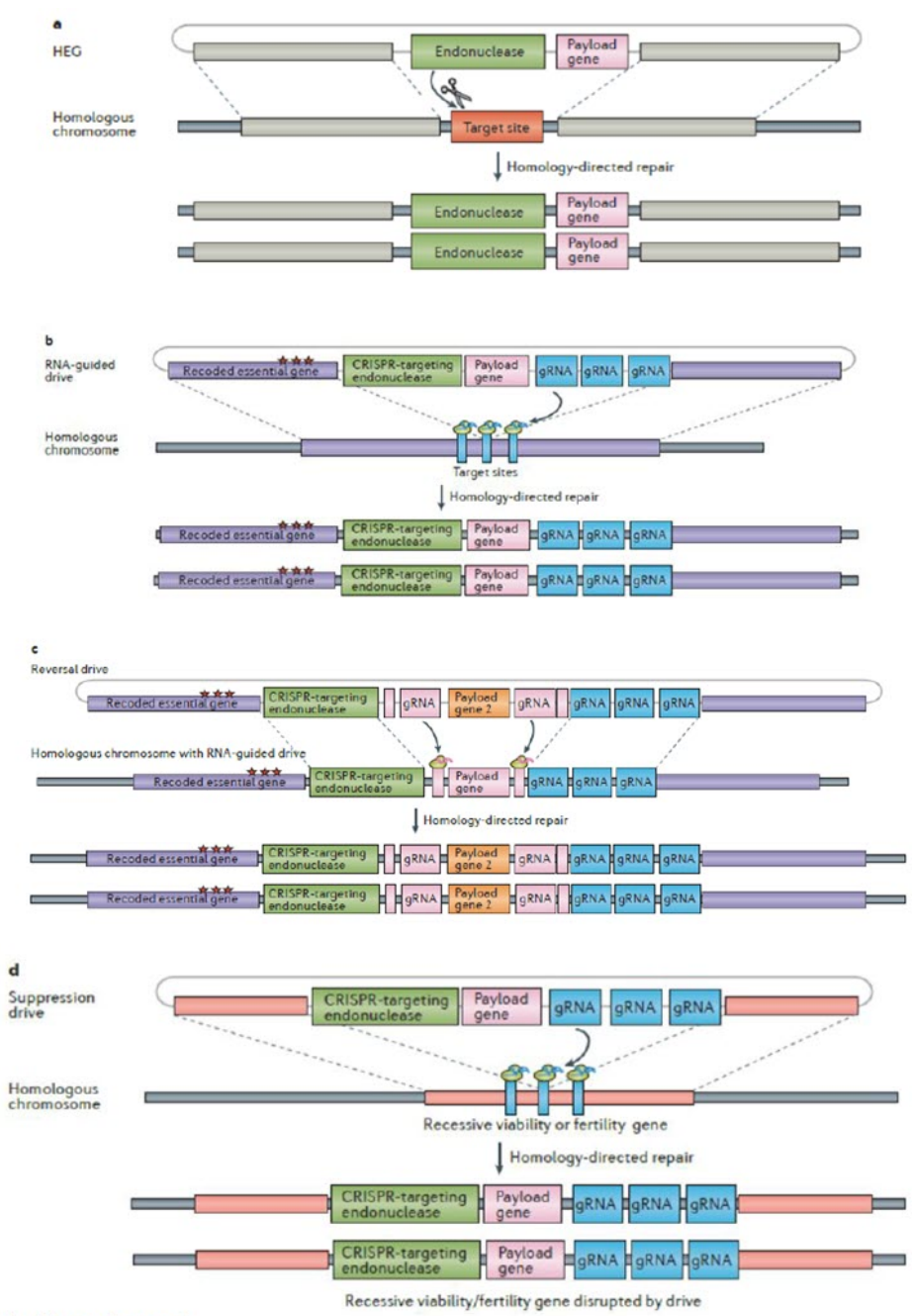

(Noble et al, 2019)

Figure 3: Shows how homing into female infertility genes can be used in mosquito population control

Homing reaction can also be used to spread ('knock-in') a novel 'cargo' gene through a population. The cargo could be an effector gene that disrupts parasite transmission through the mosquito. More than 28 effector genes can interfere to some extent with malaria parasite transmission (Wang and Jacobs-Lorena 2013), including antimicrobial peptides, single-chain antibodies, immune system activators, and peptides that bind to mosquito proteins in the midgut or salivary glands. Because malaria is transmitted only by female mosquitoes, a population-wide distortion of the sex ratio towards males would have a direct impact in reducing malaria transmission. In Aedes mosquitoes, there is a naturally occurring driving Y chromosome that in some crosses leads to more than $90 \%$ male progeny. Proof-of-principle demonstration of this route to drive in A. gambiae was reported by Galizi et al. (2014), who showed that expression of an engineered 
variant of the PpoI nuclease from a slime mold, expressed during spermatogenesis using the B2-tubulin promoter sequences, led in some lines to males producing $95 \%$ male offspring. Other sorts of gene drive systems have been proposed for spreading an effector through pest populations, including chromosomal rearrangements that display under-dominance, and various combinations of toxins and antidotes that mimic under-dominance systems, maternal effect dominant embryonic arrest (MEDEA) systems, or variants thereof (Marshall and Akbari 2016). In a MEDEA system, the progeny of heterozygous females dies unless they inherit the MEDEA element. These sorts of drivers are 'weaker' than those based on homing or driving sex chromosomes (Burt et al., 2017). However, when a single mosquito species that transmit malaria parasites is eliminated there is a possibility of a successor species arising to take its place. This species will start transmitting the malaria parasites.

Transposons should not be used to control mosquito populations. They are linked to a genetic payload which would increase the frequency of the transposable element and genetic payload in the genome of a target organism, and eventually in the population. However, transposable elements often have transposition rates that are too low to be usable. They are unpredictable owing to lack of control over their integration sites and have proven to be difficult to mobilize after integration ( Tu and Li, 2013).

Sex-linked meiotic drives have a low resistance allele generation rate. They can be reversed and cannot be removed with wild-type. They can suppress or eliminate populations. This can bring about unanticipated ecological ramifications. They have a moderate rate of spread and can result in the extinction of some species. They can thus be used to suppress or eliminate mosquito species that transmit the malaria parasites.

Supernumerary B-chromosomes have been suggested as vehicles to carry payload genes. This is because they are inherited at rates that are greater than Mendelian rates and can express transcripts. However, they are poorly understood making their engineering difficult. This makes them unfavorable for mosquito control.

The Killer-Rescue system uses a toxin and an antidote gene that are at separate loci. It is a hypothetical threshold-dependent gene drive system. The inverse $M E D E A$ system relies on a toxin that takes effect in the zygote unless it receives a maternally delivered antidote. The Merea system functions similarly to $M E D E A$, but the antidote to the maternal toxin is recessive. The Semele system, conversely, uses a paternal semen-based toxin and a maternally delivered antidote. TheMedusa system induces a population crash by utilizing a pair of sex-linked toxins and antidotes. In the future, RNA-guided nucleases may contribute to the development of each of these systems in mosquito species (Raul, 2018).

These various genetic approaches to vector control have the potential to provide several desirable features. They act to reduce disease transmission and thus can contribute to the goals of disease elimination and eradication. They are widely applicable, able to act in diverse settings, whether hypo- or holo-endemic, urban or rural, against mosquitoes that feed indoors or outdoors, during daytime or night-time, and can control mosquito populations that are otherwise difficult to access. They provide area-wide control, and therefore protection without obvious biases relating to a person's age, wealth, or education; they should be compatible with and complementary to other disease control measures, both current and underdevelopment; and they can be relatively easy to deliver and deploy, with little or no change required in how people behave, and as a result have the potential to be highly cost-effective. It is these key features that motivate the continued development of gene drive approaches to malaria control (Burt et al., 2017). 


\section{Conclusion}

Although gene drives can be useful in malaria control there is a need to employ strategies to control the spread of genetically modified mosquitoes after release. This will help prevent unintended ecological effects and keep trust in scientists (Marshall et al., 2017). This is because experiments and deterministic models have shown that drive resistance can result from mutations that block cutting by the CRISPR nuclease. The effects of this phenomenon are not always certain. However, this is not a major impediment to the invasion of unintended populations. Genetically modified mosquitoes can, however, cross international borders, even from isolated islands. Thus, there is a need to develop 'local', sensitive methods of monitoring population genetics, intrinsically self-exhausting gene drive systems and strategies for countering self-propagating drive systems as well as removing all engineered genes from wild populations (Noble et al., 2018). Several other promising gene drive systems have thus far only been advanced at the theoretical level.

There is an unknown likelihood of unauthorized releases of self-propagating gene drive systems. This is affected by species, application, containment strategies, economic motivations, drive development stages, geography and the caution of the scientists. However, the possibility of consequently having serious negative ecological consequences given the high likelihood of spread to most populations of the target species is reduced. This is because gene drive systems are typically predicted to be transient and are not designed to alter traits of the host organism or other species. Thus, there is no need for social backlash from the unauthorized spread of self-propagating gene drives (Noble et al., 2018; Funk and Rainie, 2015). However, there is a need to develop local capacity on gene drives in areas where they are to be used. This will help improve their acceptance and uptake.

\section{References}

1. Gubler, D. J. (2006) 'Human Arbovirus Infections Worldwide', Annals of the New York Academy of Sciences, 951(1), pp. 13-24. doi: 10.1111/j.1749-6632.2001.tb02681.x.

2. Derbie, H. (2019) What Good Are Mosquitoes? The Important Roles Mosquitoes Play in Our World. Available at: https://www.thoughtco.com/what-good-are-mosquitoes-1968303 (Accessed: 22 January 2020 ).

3. Crans, W. J. (2004) A classification system for mosquito life cycles: life cycle types for mosquitoes of the northeastern United States. Available at: https://pdfs.semanticscholar.org/0a2d/3db372987ef00d954a52ca42ae0ed94002e5.pdf (Accessed: 22 January 2020).

4. Marí, R. B. et al. (2012) Journal of the European Mosquito Control Association ISSN 1460-6127; www.e-m-b.org First published online 6, European Mosquito Bulletin. Available at: www.e-m-b.org (Accessed: 22 January 2020).

5. Halouzka, J. et al. (1999) 'Isolation of Borrelia afzelii from overwintering Culexpipiens biotype molestus mosquitoes', Infection. MMV MedizinVerlag GmbH, 27(4-5), pp. 275-277. doi: 10.1007/s150100050029.

6. Kindhauser, M. K. et al. (2016) 'Zika: the origin and spread of a mosquito-borne virus', Bull World Health Organ. doi: 10.2471/BLT.16.171082.

7. Lim, C. and McAleer, M. (2005) 'Ecologically sustainable tourism management', Environmental Modelling and Software. Elsevier BV, 20(11), pp. 1431-1438. doi: 10.1016/j.envsoft.2004.09.023.

8. Lundkvist, E. et al. (2003) 'Diving beetles (Dytiscidae) as predators of mosquito larvae (Culicidae) in field experiments and in laboratory tests of prey preference', Bulletin of Entomological Research. Cambridge 
University Press (CUP), 93(3), pp. 219-226. doi: 10.1079/ber2003237.

9. Quiroz-Martínez, H. and Rodríguez-Castro, A. (2007) 'AQUATIC INSECTS AS PREDATORS OF MOSQUITO LARVAE', Journal of the American Mosquito Control Association. The American Mosquito Control Association, 23(sp2), pp. 110-117. doi: 10.2987/8756-971x(2007)23[110:aiapom]2.0.co;2.

10. Rueda, L. M. (2004) Pictorial keys for the identification of mosquitoes (Diptera: Culicidae) associated with Dengue Virus Transmission. Available at: http://www.mapress.com/zootaxa/ (Accessed: 22 January 2020).

11. Schneider, B. S. and Higgs, S. (2008) 'The enhancement of arbovirus transmission and disease by mosquito saliva is associated with modulation of the host immune response', Transactions of the Royal Society of Tropical Medicine and Hygiene, 102(5), pp. 400-408. doi: 10.1016/j.trstmh.2008.01.024.

12. Stark, K. R. and James, A. A. (1998) 'Isolation and characterization of the gene encoding a novel factor Xa- directed anticoagulant from the yellow fever mosquito, Aedesaegypti', Journal of Biological Chemistry, 273(33), pp. 20802-20809. doi: 10.1074/jbc.273.33.20802.

13. Thien, L. B. (1969) 'MOSQUITO POLLINATION OF HABENARIA OBTUSATA (ORCHIDACEAE)', American Journal of Botany, 56(2), pp. 232-237. doi: 10.1002/j.1537-2197.1969.tb07528.x.

14. Waldbauer, G. (1998) The handy bug answer book. Visible Ink Press. Available at: http://agris.fao.org/agris-search/search.do?recordID=US201300067714 (Accessed: 22 January 2020).

15. Weaver, S. C. and Lecuit, M. (2015) 'Chikungunya virus and the global spread of a mosquito-borne disease', New England Journal of Medicine. Massachussetts Medical Society, pp. 1231-1239. doi: 10.1056/NEJMra1406035.

16. Collins, J. P. (2018) 'Gene drives in our future: Challenges of and opportunities for using a self-sustaining technology in pest and vector management', BMC Proceedings. BioMed Central Ltd. doi: 10.1186/s12919018-0110-4.

17. Champer, J., Buchman, A. and Akbari, O. S. (2016) 'Cheating evolution: engineering gene drives to manipulate the fate of wild populations', Nature Publishing Group. doi: 10.1038/nrg.2015.34.

18. Burt, A. et al. (2018) 'Gene drive to reduce malaria transmission in sub-Saharan Africa', Journal of Responsible Innovation, 5(sup1), pp. S66-S80. doi: 10.1080/23299460.2017.1419410.

19. Neves, M. P. and Druml, C. (2017) 'Ethical implications of fighting malaria with CRISPR/Cas9', BMJ Global Health. BMJ Publishing Group. doi: 10.1136/bmjgh-2017-000396.

20. Hartley, S. et al. (2019) 'Knowledge engagement in gene drive research for malaria control Introduction: Development of gene drive mosquitoes'. doi: 10.1371/journal.pntd.0007233.

21. Sackler Forum: Trends in synthetic biology and gain of function and regulatory implications | Royal Society (2016). Available at: https://royalsociety.org/topics-policy/publications/2016/sackler-forum-report/ (Accessed: 23 January 2020).

22. Leitschuh, C. M. et al. (2018) 'Developing gene drive technologies to eradicate invasive rodents from islands', Journal of Responsible Innovation, 5, pp. 121-138. doi: 10.1080/23299460.2017.1365232.

23. Medina, R. F. (2017) 'Journal of Responsible Innovation Gene drives and the management of agricultural pests'. doi: 10.1080/23299460.2017.1407913.

24. Hayirli, T. C. and Martelli, P. F. (2019) 'Gene drives as a response to infection and resistance', Infection and Drug Resistance. Dove Medical Press Ltd., 12, pp. 229-234. doi: 10.2147/IDR.S187424.

25. James, S. et al. (2018) 'Pathway to Deployment of Gene Drive Mosquitoes as a Potential Biocontrol Tool for Elimination of Malaria in Sub-Saharan Africa: Recommendations of a Scientific Working Group +', Am. J. Trop. Med. Hyg, 98, pp. 1-49. doi: 10.4269/ajtmh.18-0083. 
26. Ashley, E. A. et al. (2014) 'Spread of Artemisinin Resistance in Plasmodium falciparum Malaria', New England Journal of Medicine, 371(5), pp. 411-423. doi: 10.1056/NEJMoa1314981.

27. Menard, D. et al. (2016) 'A Worldwide Map of Plasmodium falciparum K13-Propeller Polymorphisms', New England Journal of Medicine, 374(25), pp. 2453-2464. doi: 10.1056/NEJMoa1513137.

28. Imwong, M. et al. (2017) 'The spread of artemisinin-resistant Plasmodium falciparum in the Greater Mekong subregion: a molecular epidemiology observational study', The Lancet Infectious Diseases. doi: 10.1016/S1473-3099(17)30048-8.

29. Barnes, K. G. et al. (2017) 'Genomic Footprints of Selective Sweeps from Metabolic Resistance to Pyrethroids in African Malaria Vectors Are Driven by Scale up of Insecticide-Based Vector Control'. doi: 10.1371/journal.pgen.1006539.

30. Isaacs, A. T. et al. (no date) 'Transgenic Anopheles stephensicoexpressing single-chain antibodies resist Plasmodium falciparum development'. doi: 10.1073/pnas.1207738109/-/DCSupplemental.

31. Bhatt, S. et al. (2015) 'The effect of malaria control on Plasmodium falciparum in Africa between 2000 and 2015', Nature. Nature Publishing Group, 526(7572), pp. 207-211. doi: 10.1038/nature15535.

32. WHO (2012) WHO global malariaprogramme global plan for insecticide resistance management in malaria vectors WHO Library Cataloguing-in-Publication Data Global plan for insecticide resistance management in malaria vectors (GPIRM). Available at: http://www.who.int/about/licensing/copyright_form/en/index.html (Accessed: 23 January 2020).

33. Pike, A. et al. (2017) Changes in the microbiota cause genetically modified Anopheles to spread in a population Downloaded from, Science. Available at: http://science.sciencemag.org/ (Accessed: 23 January 2020).

34. Swale, D. R. et al. (2016) 'An insecticide resistance-breaking mosquitocide targeting inward rectifier potassium channels in vectors of Zika virus and malaria', Scientific Reports. Nature Publishing Group, 6. doi: $10.1038 /$ srep36954.

35. Bian, G. et al. (2013) 'Wolbachia invades Anopheles stephensi populations and induces refractoriness to Plasmodium infection', Science. American Association for the Advancement of Science, 340(6133), pp. 748-751. doi: 10.1126/science.1236192.

36. Lovett, B. et al. (2019) 'Transgenic Metarhizium rapidly kills mosquitoes in a malaria-endemic region of Burkina Faso', Science. American Association for the Advancement of Science, 364(6443), pp. 894-897. doi: $10.1126 /$ science.aaw8737.

37. Wang, S. and Jacobs-Lorena, M. (2013) 'Genetic approaches to interfere with malaria transmission by vector mosquitoes', Trends in Biotechnology, pp. 185-193. doi: 10.1016/j.tibtech.2013.01.001.

38. Carvalho, D. O. et al. (2015) 'Suppression of a Field Population of Aedesaegypti in Brazil by Sustained Release of Transgenic Male Mosquitoes', PLOS Neglected Tropical Diseases. Edited by K. E. Olson, 9(7), p. e0003864. doi: 10.1371/journal.pntd.0003864.

39. Esvelt, K. M. et al. (2014) 'Concerning RNA-guided gene drives for the alteration of wild populations', eLife. eLife Sciences Publications Ltd, 3(July2014), pp. 1-21. doi: 10.7554/eLife.03401.

40. Noble, C. et al. (2018) 'Current CRISPR gene drive systems are likely to be highly invasive in wild populations', eLife. eLife Sciences Publications Ltd, 7. doi: 10.7554/eLife.33423.

41. Marshall, J. M. et al. (no date) 'Overcoming evolved resistance to population-suppressing homing-based gene drives OPEN'. doi: 10.1038/s41598-017-02744-7.

42. Funk C, R. L. (no date) (PDF) Public and Scientists' Views on Science and Society. Available 
at: https://www.researchgate.net/publication/279513537_Public_and_Scientists'_Views_on_Science_and_Society (Accessed: 23 January 2020).

43. National Academies of Sciences, Engineering, and Medicine, 2016. Gene drives on the horizon: advancing science, navigating uncertainty, and aligning research with public values. National Academies Press.

44. Eckhoff, P. A. et al. (no date) 'Impact of mosquito gene drive on malaria elimination in a computational model with explicit spatial and temporal dynamics'. doi: 10.1073/pnas.1611064114.

45. Kyrou, K. et al. (2018) 'A CRISPR-Cas9 gene drive targeting double sex causes complete population suppression in caged Anopheles gambiae mosquitoes', Nature biotechnology. NLM (Medline), 36(11), pp. 1062-1066. doi: 10.1038/nbt.4245.

46. Medina, R. F. (2018) 'Gene drives and the management of agricultural pests', Journal of Responsible Innovation. Routledge, 5, pp. S255-S262. doi: 10.1080/23299460.2017.1407913.

47. Beaghton, A., Beaghton, P. J. and Burt, A. (2016) 'Gene drive through a landscape: Reaction-diffusion models of population suppression and elimination by a sex ratio distorter', Theoretical Population Biology. Academic Press Inc., 108, pp. 51-69. doi: 10.1016/j.tpb.2015.11.005.

48. Marshall, J. M. et al. (2017) 'Overcoming evolved resistance to population-suppressing homing-based gene drives. Scientific Reports. doi: 10.1038/s41598-017-02744-7.

49. Čartolovni, A. (2017) 'Teilhard de Chardin's oeuvre within an ongoing discussion of a gene drive release for public health reasons'. Life Sciences Society and Policy. doi: 10.1186/s40504-017-0064-8.

50. Noble, C., Min, J., Olejarz, J., Buchthal, J., Chavez, A., Smidler, A. L., DeBenedictis, E. A., Church, G. M., Nowak, M. A., Esvelt, K. M. (2019). Daisy-chain gene drives for the alteration of local populations. Proceedings of the National Academy of Sciences. 116 (17) 8275-8282; DOI: 10.1073/pnas.1716358116

51. Scudellari, M. 2019. Self-destructing mosquitoes and sterilized rodents: the promise of gene drives. Nature. News feature.

52. Tu Z and Li S. (2013). Mobile Genetic Elements of Malaria Vectors and Other Mosquitoes. In: Madame Curie Bioscience Database [Internet]. Austin (TX): Landes Bioscience; 2000-2013. Available from: https://www.ncbi.nlm.nih.gov/books/NBK6186/

53. Chen L, He Z, Qin L, Li Q, Shi X, et al. (2011) Antitumor Effect of Malaria Parasite Infection in a Murine Lewis Lung Cancer Model through Induction of Innate and Adaptive Immunity. PLoS ONE 6(9): e24407. doi:10.1371/journal.pone.0024407

54. Yang Y., Liu Q., Lu J., Adah D., Yu S., Zhao S., Yao Y., Qin L., Qin L. and Chen X. 2017. Exosomes from Plasmodium-infected hosts inhibit tumor angiogenesis in a murine Lewis lung cancer model. Oncogenesis. 6, e351; doi:10.1038/oncsis.2017.52 